\title{
Reconciling calculated and measured adsorption enthalpies for oxyanions on rutile (110)
}

\author{
F.T.ADAMS ${ }^{1}$, KAREN PHAN $^{2}$, J.D.KUBICKI ${ }^{3}$ AND N.KABENGI
}

${ }^{1}$ Georgia State University, Department of Geosciences, Atlanta, GA, 30303 USA (fadams4@student.gsu.edu)

${ }^{2}$ Georgia State University, Department of Chemistry, Atlanta, GA, 30303, USA (kphan12@student.gsu.edu)

${ }^{3}$ University of Texas at El Paso, Department of Geological

Sciences, El Paso, TX 79968 (jdkubicki@utep.edu)

* correspondence: (kabengi@gsu.edu)

Attempts to reconcile predicted adsorption energies with those measured experimentally have not always been successful. Several possible factors can account for previously observed discrepancies, namely, the use of powdered samples in experiments while modeling and calculations are performed for a single face or even single sites on a particular face, and the energetic heterogeneity of various surface sites and defects (radical sites, step corners, regular terraces) that is not well captured. In an attempt to further explore the possibility of converging on adsorption enthalpies, this work will compare calculated adsorption enthalpies to experimental values on rutile nanoparticles. The rutile-water interface and, in particular, the predominant (110) surface has been extensively studied and is often considered the archetypical surface of metal oxide-fluid interface investigations. It is ideal for both experimental and computational work in that: i) a rutile nanopowder dominated by the (110) surface is available from Tioxide Corporation and ii) atomistically-detailed models of the (110) singlecrystal surface with various terminations are available. In this sense, the efforts can focus on the computational approach as a source of error in the adsorption energy calculations. We will study the adsorption and exchange of carbonate, phosphate, chromate and oxalate in a background of $\mathrm{NaCl}$ and $\mathrm{NaNO}_{3}$ solutions. Measurements of $\Delta \mathrm{H}_{\text {ads }}$ will be made using flow microcalorimeters and DFT calculations will be performed on model systems where full solvation of the surface and oxyanions were included. An additional benchmarking will be made against measurements made on anatase as it offers significantly higher specific surface areas, and potential, surface sites. The key advantage of this work is the real-time collaboration between the experimental and computational work on a well-characterized mineral surface. 